\title{
COVID-19 in an Intrauterine Growth Restriction (IUGR) Infant with Congenital Heart Disease: Case Report and Literature Review
}

\author{
Mostafa Elbehery $^{1}$, Farid A. Munshi ${ }^{1}$, Abdullah Alzahrani ${ }^{2}$, Mohammed Bakhsh ${ }^{2}$, Majied Alariefy ${ }^{3}$ \\ 1. Pediatric Cardiac Critical Care Unit, King Faisal Cardiac Center, King Abdulaziz Medical City, Jeddah, SAU 2. \\ Pediatric Intensive Care, King Abdulaziz Medical City, Ministry of National Guard - Health Affairs, Jeddah, SAU 3. \\ Pediatric Cardiology, King Abdulaziz Medical City, Ministry of National Guard - Health Affairs, Jeddah, SAU
}

Corresponding author: Farid A. Munshi, munshifa@ngha.med.sa

\begin{abstract}
In pediatrics, reported coronavirus disease-19 (COVID-19) cases showed that this category of patients usually presented with no symptoms or mild to moderate COVID-19 disease. However, infants less than one-year-old had a more severe presentation or even critical condition and respiratory failure. Patients with chronic disease and congenital heart disease (CHD) may have serious effects on course of COVID-19 in neonate and early infancy. There is very limited data about confirmed COVID-19 cases with CHD in neonates and early infancy. We report our case with confirmed COVID-19 diagnosed in the neonatal period with multiple ventricular septal defects (VSDs) and patent ductus arteriosus (PDA) who presented with respiratory distress and respiratory acidosis.
\end{abstract}

Categories: Cardiology, Pediatrics, Infectious Disease

Keywords: covid-19, pediatric congenital heart disease, intrauterine growth retardation

\section{Introduction}

The infection with coronavirus disease 2019 (COVID-19) (severe acute respiratory syndrome coronavirus 2 (SARS-CoV-2)) has been increasing rapidly since it was identified for the first time in December 2019 in Wuhan, China [1-2]. As of March 2020, it was declared a global pandemic by the World Health Organization (WHO).

Although all age groups are susceptible, those with comorbidities are more likely to have more complications and to become severely ill [3]. In a study that described the epidemiological characteristics of SARS-CoV-2 in pediatrics, over $90 \%$ were asymptomatic or presented with mild to moderate symptoms [4]. However, infants under one year showed the highest proportion of severe and critical cases, about $10.6 \%$ compared to other age groups suggesting that infants may be at higher risk of severe respiratory failure [4]. Moreover, the presence of congenital heart diseases (CHDs) or any other comorbidity may have a serious effect on the course of this disease in the neonates and early infancy. There is limited data about the effect of SARS-CoV-

Received 08/29/2020

Review began 09/01/2020 Review ended 09/06/2020 Published 09/07/2020

\section{() Copyright 2020}

Elbehery et al. This is an open access article distributed under the terms of the Creative Commons Attribution License CC-BY 4.0., which permits unrestricted use, distribution, and reproduction in any medium, provided the original author and source are credited.
2 in pediatrics with CHDs especially in the age under one year.

\section{Case Presentation}

Here, we report a case of a six-week-old female infant, who was diagnosed upon birth with intrauterine growth restriction (IGUR), CHD (multiple ventricular septal defects (VSDs) and patent ductus arteriosus (PDA)), and neonatal cholelithiasis, who presented with symptomatic COVID-19.

Regarding this infant's past history, she was diagnosed upon birth with symmetrical IUGR with a bodyweight of $2.095 \mathrm{~kg}$; neonatal cholelithiasis with direct hyperbilirubinemia for which she was commenced on ursodeoxycholic acid and multivitamins, and CHD in form of multiple VSDs and small PDA but she was not on any anti failure medications. It is worth mentioning that TORCH (Toxoplasma gondii, Rubella, Cytomegalovirus (CMV) and the Herpes Simplex Virus) screening was done during the neonatal period and came negative.

At the age of 40-days, the patient presented to the emergency department with a four-day history of dry cough, rhinorrhea, and shortness of breathing. Acute respiratory infection (ARI) screening was done as per protocol and the total score was 5 which means that the infant is a possible COVID-19 patient. Moreover, the parents were asymptomatic and both had been tested for COVID-19 which turned out to be negative; however, her both grandparents were tested positive for COVID-19 and were admitted to the ICU.

On examination, the patient had a low-grade fever, tachycardia, tachypnea, subcostal retractions, clear chest on auscultation apart from transmitted sounds, and a pan-systolic murmur 3/6. There was no sacral edema and no hepatomegaly. A chest X-ray was done which revealed mild prominence of cardiomediastinal 


\section{Cureus}

contour and pulmonary vasculature (Figure 1). Venous blood gas was done and showed uncompensated respiratory acidosis. Furthermore, viral screening and laboratory tests were sent and reported as shown in Table 1.

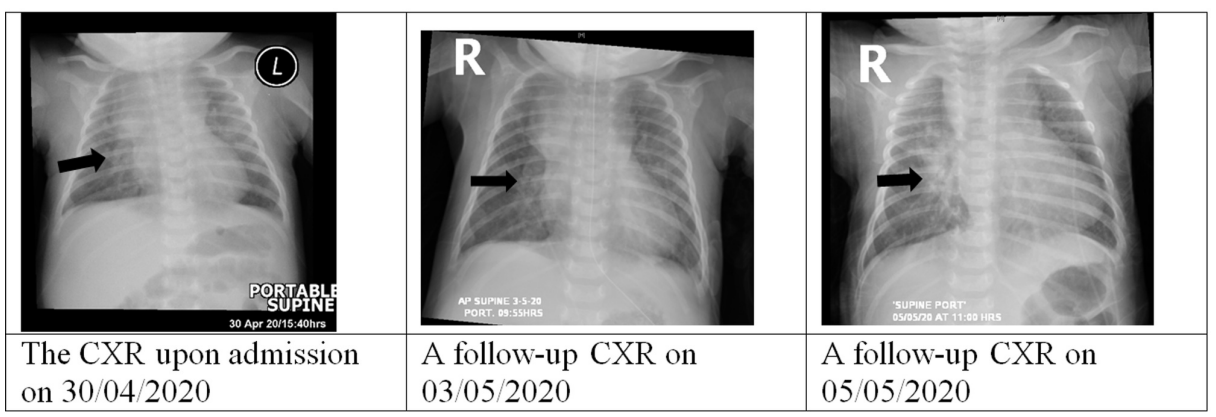

FIGURE 1: Serial chest X-ray (CXR) studies during the admission

Patient Characteristics

Gestational age

37 weeks

Age at presentation

40 days

Gender

Female

Mode of delivery

Vaginal

APGAR (1,5 min)

9 and 9

Birth weight

$2.095 \mathrm{Kg}$.

Weight at presentation

$3.29 \mathrm{Kg}$.

Vital signs on admission

Heart rate

$165 / \min$

Blood pressure

$97 / 50 \mathrm{mmHg}$

Temperature

$37.7^{\circ} \mathrm{C}$

Respiratory rate

$59 / \min$

Oxygen saturation on room air

$96 \%$

Oxygen saturation on nasal cannula $2 \mathrm{~L} / \mathrm{M}$

$100 \%$

Laboratory values

Complete blood count

White blood cells $\left(\times 10^{3} / \mu \mathrm{L}\right)$

Neutrophils (\%)

Lymphocytes (\%)

28

Hemoglobin $(g / d L)$

Platelets $\left(\times 10^{3} / \mu \mathrm{L}\right)$

Biochemistry and cardiac markers

Blood urea nitrogen (mmol/L)

Creatinine (umol/L) 


\section{Cureus}

AST (IU/L)

Total bilirubin (umol/L)

Direct bilirubin (umol/L)

CRP (mg/L)

Procalcitonin (ug/L)

D-Dimer (mg/L)

LDH (U/L)

Ferritin (ug/L)

ESR $(\mathrm{mm} / \mathrm{hr})$

CK (IU/L)

Troponin I (pg/mL)

Virology and microbiology

Blood culture

Urine culture

Negative

Stool culture

Negative

Respiratory viral panel

Negative

EBV (PCR)

Not detected

EBV-IgM

Negative

EBV-IgG (AU/ml)

$<10$

CMV-IgM

Negative

CMV-lgG (U/ml)

129

COVID-19 (rRT PCR)

Detected

Venous blood gas

$\mathrm{pH}$

pCO2

\section{TABLE 1: Patient characteristics, vital signs on admission and laboratory values}

APGAR: Appearance, Pulse, Grimace, Activity, and Respiration; ALT: Alanine aminotransferase; AST: Aspartate aminotransferase; CRP: C-reactive protein; LDH: lactate dehydrogenase; ESR: erythrocyte sedimentation rate; CK: Creatine kinase; EBV: Epstein-Barr virus; PCR: polymerase chain reaction; IgM: Immunoglobulin M; IgG: Immunoglobulin G; rRT PCR: real-time reverse transcription-polymerase chain reaction; pCO2: partial pressure of carbon dioxide;

HCO3: Bicarbonate; BE: Barium enema.

In light of the high possibility of positive SARS-CoV-2, the patient was admitted to a negative pressure room in the pediatric cardiac intensive care unit (PCICU) with enhanced infection control precautions requiring an N95 mask, eye shield, gloves, and gowns. Later on, SARS-CoV-2 test (real-time reverse transcriptionpolymerase chain reaction (rRT PCR)) was reported positive; therefore, the pediatric COVID team was involved in the management as per hospital protocol.

In terms of the management, she was given a STAT dose of furosemide in the emergency room, and oxygen 
support via nasal cannula was commenced. After admitting her to the PCICU, the supportive and therapeutic care began. She was kept nil per os (NPO) on proper fluid therapy, oxygen support continued via nasal cannula $2 \mathrm{~L} / \mathrm{M}$, and she has commenced on regular anti failure medications in the form of furosemide and captopril along. Acetaminophen was given when needed for fever. Antibiotics and antiviral were not started as recommended by the pediatric COVID team who preferred to monitor her condition and to commence only if her condition deteriorated. ECG was done during her stay in PCICU and showed normal sinus rhythm.

Later on, her following blood gases showed a gradual improvement of the hypoventilation. Her condition showed substantial improvement within the next three days as the fever subsided, her respiratory distress signs resolved, the tachycardia improved, the oxygen support was weaned off and the feeding was started orally (Figure 2). Moreover, the following laboratory results were generally improving. Although C-reactive protein (CRP) and procalcitonin slightly increased, the patient remained stable hemodynamically and showed no signs of sepsis; therefore, no antibiotics were started and blood and urine cultures were sent and reported negative. The COVID-19 rRT PCR test, which was repeated every 3-5 days, remained positive on six occasions before being reported negative after 28 days, and she was discharged home in a good condition on anti failure medications.

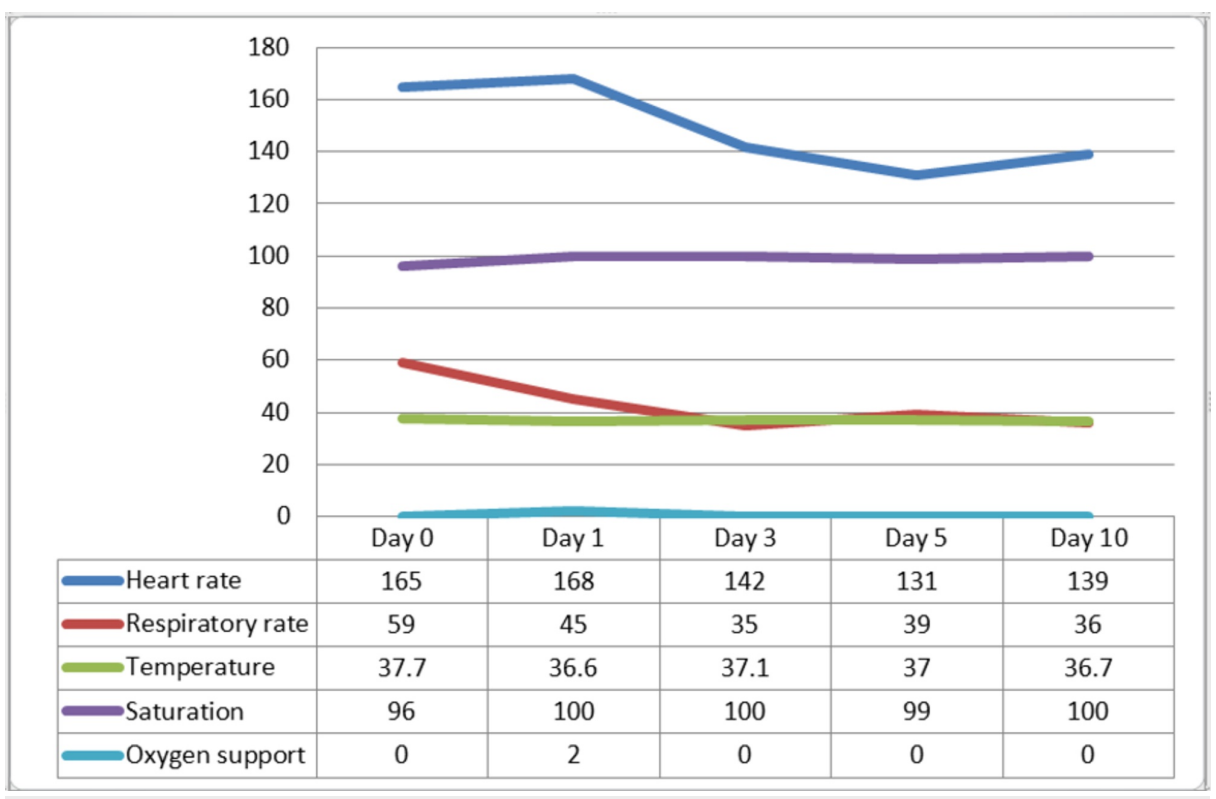

FIGURE 2: Vital signs chart

The family was contacted after discharge by phone (virtual clinic) and the baby was doing fine with no issues.

\section{Discussion}

In this case, there was no direct contact with positive cases; however, her father, who was tested negative, had direct contact with the grandfather who turned out later to be positive. Most of the children with SARSCoV-2 were identified through contact tracing in the households of infected adults [2]. Furthermore, all case reports of COVID-19 in children confirm household or family cluster transmission [5-7].

Generally, the infection by COVID-19 among children is low; around 1.7\% of all COVID-19 infections in the USA are under 18 years old, while it is around $2.4 \%$ in China $[2,8]$. The infants were $11.8 \%$ of the whole infections under 18 years old [4]. The clinical manifestations of the disease tend to be mild in children comparing with adults [2]. According to a recent study that reviewed the epidemiological characteristics of 2143 children with COVID-19, over $90 \%$ of them were asymptomatic, mild, or moderate cases [4]. The common symptoms of COVID-19 are fever, cough, and shortness of breath which is similar to this case [9]. Although the presence of comorbidities might alter the course of the disease to be more severe, our patient, who was known to have CHD and IGUR, did not have any complications and fully recovered within few days [8].

In this case, the patient's symptoms and signs improved on the third day of admission; however, the rRT PCR test remained positive for 28 days and that was similar to a case report of a 14-month-old infant in China [10]. Since the infectivity of the patient was uncertain, the patient was kept in the hospital until she had a negative rRT PCR result. In a joint paper published by the National Centre for Infectious Diseases (NCID) and the Chapter of Infectious Disease Physicians, Academy of Medicine, Singapore, the contagious period of 
SARS-CoV-2 may start two days before the onset of symptoms, and continues for about 7-10 days after the onset of symptoms [11]. Furthermore, there was no viable SARS-CoV-2 detected after 14 days of the symptoms even with positive PCR tests [11].

To date, there are no data on COVID-19 in pediatric patients with CHDs. Therefore, the current management strategies are customized according to the patient's condition along with what is known about the effect of COVID-19 in pediatric patients. Because of the limited available data, it is challenging to come up with any definitive recommendations or specific management guidelines for pediatric patients with CHDs.

The SARS-CoV-2 virus enters the cells by using angiotensin converting enzyme (ACE)2 as a receptor, and since ACE2 is expressed in many tissues including the cardiac tissue, there have been some hypotheses that the use of ACE-inhibitors and angiotensin receptor 1 blocker (ARBs) may have an effect on the course of COVID-19 [12-14]. In this case, we started ACE inhibitors as a part of the anti failure measures; however, there was no change in the expected course of the disease, but the ACE2 protein is affected by developmental factors, and it is possible that the ACE2 protein in children has a lower binding affinity to the SARS-CoV-2 virus, or that the intracellular responses induced by ACE2 in alveolar epithelial cells are milder in children when compared to adults, therefore, more studies are needed before making any recommendations regarding the usage of ACE-inhibitor and ARB medications in COVID-19 patients [14].

\section{Conclusions}

Most children contract the infection via horizontal transmission from a household member; therefore, families should take precautions to protect their children. Cough, respiratory distress, and fever are the most common presenting symptoms; however, the disease is milder in pediatrics comparing with adult patients. Despite the rRT PCR test remaining positive for a longer time, the discharge decision can be made based on the clinical condition as long as the patient improved and was properly isolated for at least 10-14 days after the onset of the symptoms. Moreover, more studies are needed in pediatric COVID-19 patients with CHDs and the effect of ACE inhibitors and ARB medications before developing any recommendations regarding the management of those patients.

\section{Additional Information}

\section{Disclosures}

Human subjects: Consent was obtained by all participants in this study. King Abdullah International Medical Research Center issued approval RJ20/141/J. Approved by King Abdullah International Medical Research Center Institutional Review Board; Study number: RJ20/141/J. Approval date 19/08/2020. Conflicts of interest: In compliance with the ICMJE uniform disclosure form, all authors declare the following: Payment/services info: All authors have declared that no financial support was received from any organization for the submitted work. Financial relationships: All authors have declared that they have no financial relationships at present or within the previous three years with any organizations that might have an interest in the submitted work. Other relationships: All authors have declared that there are no other relationships or activities that could appear to have influenced the submitted work.

\section{Acknowledgements}

We gratefully acknowledge Dr. Rahaf Waggass and Dr. Waheed Raedi for their valuable contribution to this case study.

\section{References}

1. Zhu N, Zhang D, Wang W, Li X, Yang B, Song J, Zhao X: China novel coronavirus investigating and research team: a novel coronavirus from patients with pneumonia in China, 2019. N Engl J Med. 2020, 382:727-33. 10.1056/nejmoa2001017

2. WHO: report of the WHO-China Joint Mission on coronavirus disease 2019 (COVID-19) . (2020). Accessed: July 10, 2020: https://www.who.int/docs/default-source/coronaviruse/who-china-joint-mission-on-covid19-final-report.pdf.

3. Chen ZM, Fu JF, Shu Q, et al.: Diagnosis and treatment recommendations for pediatric respiratory infection caused by the 2019 novel coronavirus. World J Pediatr. 2020, 16:240-246. 10.1007/s12519-020-00345-5

4. Dong Y, Mo X, Hu Y, Qi X, Jiang F, Jiang Z, Tong S: Epidemiology of COVID-19 among children in China . Pediatrics. 2020, 145:e20200702. 10.1542/peds.2020-0702

5. Cai JH, Wang XS, Ge YL, et al.: First case of 2019 novel coronavirus infection in children in Shanghai [Article in Chinese]. Zhonghua Er Ke Za Zhi. 2020, 58: 10.3760/cma.j.issn.0578-1310.2020.0002

6. Chan JF, Yuan S, Kok KH, et al.: A familial cluster of pneumonia associated with the 2019 novel coronavirus indicating person-to-person transmission: a study of a family cluster. Lancet. 2020, 395:514-523. 10.1016/S0140-6736(20)30154-9

7. Wei M, Yuan J, Liu Y, Fu T, Yu X, Zhang ZJ: Novel coronavirus infection in hospitalized infants under 1 year of age in China. JAMA. 2020, 323:1313-1314. 10.1001/jama.2020.2131

8. CDC COVID-19 Response Team: Coronavirus disease 2019 in children - United States, February 12-April 2, 2020. MMWR Morb Mortal Wkly Rep. 2020, 69:422-426. 10.15585/mmwr.mm6914e4

9. Zimmermann P, Curtis N: Coronavirus infections in children including COVID- 19: an overview of the 


\section{Cureus}

epidemiology, clinical features, diagnosis, treatment and prevention options in children. Pediatr Infect Dis J. 2020, 39:355-368. 10.1097/INF.0000000000002660

10. Mao L, Xu J, Xu Z, et al.: A child with household transmitted COVID-19. BMC Infect Dis. 2020, 20:329. 10.1186/s12879-020-05056-W

11. Position statement from the National Centre for Infectious Diseases and the Chapter of Infectious Disease Physicians, Academy of Medicine, Singapore - 23 May. (2020). Accessed: July 10, 2020: https://www.ams.edu.sg/view-pdf.aspx? file=media\%5C5556 fi 331.pdf\&ofile=Period + of + Infectivity+Position+Statement + (fin....

12. Wan Y, Shang J, Graham R, Baric RS, Li F: Receptor recognition by the novel coronavirus from Wuhan: an analysis based on decade-long structural studies of SARS coronavirus. J Virol. 2020, 94:e00127. 10.1128/JVI.00127-20

13. Jia HP, Look DC, Shi L, et al.: ACE2 receptor expression and severe acute respiratory syndrome coronavirus infection depend on differentiation of human airway epithelia. J Virol. 2005, 79:14614-14621. 10.1128/JVI.79.23.14614-14621.2005

14. Gurwitz D: Angiotensin receptor blockers as tentative SARS-CoV-2 therapeutics . Drug Dev Res. 2020, 81:537-540. 10.1002/ddr.21656 